\title{
Phase Transitions of Colloidal Monolayers in Periodic Pinning Arrays
}

\author{
Konrad Mangold, Paul Leiderer, and Clemens Bechinger \\ Physics Department, University of Konstanz, D-78457 Konstanz, Germany
}

(Received 23 October 2002; published 17 April 2003)

\begin{abstract}
We study the phase behavior of two-dimensional paramagnetic colloidal systems on square pinning arrays, the latter being created by a holographic optical tweezer technique. When the particle interaction strength is decreased, a transition from an incommensurate to a commensurate solid is observed. At even smaller pair potentials, the interstitial particles start to melt, whereas the particles at the substrate pinning sites are still localized. Our results are in good agreement with recent numerical studies on vortex melting in periodic pinning arrays.
\end{abstract}

PACS numbers: 82.70.Dd, 64.70.Dv, 64.70.Rh

Properties of two-dimensional (2D) systems are well known to be crucially affected by the presence of periodic substrate pinning sites. One intriguing example is a type II superconductor in the mixed state where vortices are arranged in a flux-line lattice. In the case of regularly ordered substrate pinning sites, strong anomalies in the temperature and magnetic-field dependencies of the magnetization, electrical resistance, and critical current are observed [1,2]. Those anomalies are particularly pronounced at matching fields where the number of flux lines coincides with the number of the pinning sites and essentially all of the magnetic field inside the film resides in quantized fluxoids centered on the artificial pinning sites. Several groups investigated recently the melting behavior of such vortex lattices in square, triangular, and kagome pinning lattices by means of molecular dynamics and Langevin dynamics simulations [3,4]. For weak pinning and matching field conditions, on square lattices, an order-order transition from a commensurate square vortex array to a triangular floating solid phase was observed. In contrast, for strong pinning, only a single transition from the square pinned lattice to the liquid state was found. Under nonmatching field conditions and strong substrates a multistage melting process was observed in which vortex motion takes place by an exchange process of interstitial and pinned vortices [3].

Another example where pinning effects play an important role is the phase behavior of adsorbates interacting with periodic substrates. This is important for a wide variety of condensed matter systems including, e.g., physisorbed atomic or molecular monolayers on crystalline surfaces [5-8] or colloidal particles interacting with patterned surfaces [9-12]. In all cases, the detailed understanding of how periodic substrate pinning sites modify the properties of a $2 \mathrm{D}$ system remains a sophisticated task because it is the delicate interplay between the adsorbate-adsorbate interaction and the adsorbate-substrate interaction which leads to a complex phenomenology.

In this Letter we investigate the phase behavior of a colloidal monolayer of superparamagnetic particles on a square patterned substrate potential, the latter being produced by a holographic optical tweezer technique [13]. Because the colloidal pair potential can be precisely adjusted by an external magnetic field $B$, this allows us - contrary to previous work - to vary both the substrate strength and the interparticle potential independent from each other. While on flat substrates the colloidal particles arrange in a triangular lattice at sufficiently high $B$, the presence of a square substrate potential leads to a much more complex phase behavior. Upon changing the colloidal pair potential we observe a transition from an incommensurate crystal, where only half of the pinning sites are occupied by particles, to a commensurate solid, where all pinning sites are filled. At very small pair potentials we observe delocalization of the interstitial particles while the particles at the pinning lattice sites are still highly localized. These observations are in good agreement with numerical simulations for vortex lattice melting on square pinning arrays [3]. In addition, we found a new feature, i.e., that stronger substrate potentials can also lead to a delocalization of interstitial particles. This phenomenon is assumed to be caused by particle fluctuations.

We used aqueous suspensions of superparamagnetic colloidal particles with $4.55 \mu \mathrm{m}$ diameter (Dynal company), and stabilized them with $2 \mathrm{mg} / \mathrm{ml} \mathrm{SDS}$ (sodiumdodecylsulfate) to avoid irreversible sticking at contact. The suspension was confined between two glass plates separated by a $2 \mathrm{~mm}$ thick $O$ ring. The lower plate was coated by a $0.5 \mu \mathrm{m}$ thick film of poly(methylmethacrylate) to provide a homogeneous and smooth substrate. Before the cell was assembled, the substrate was heated to about $150^{\circ} \mathrm{C}$ and a copper mesh of $15 \mu \mathrm{m}$ thickness was firmly pressed onto the substrate. This yielded several independent hexagonal-shaped hard wall compartments with an area of about $0.14 \mathrm{~mm}^{2}$ each. When the colloidal suspension was added to the cell, the particles immediately started to sediment towards the bottom plate where-owing to their high density $\left(\rho=1.7 \mathrm{~g} / \mathrm{cm}^{3}\right)$ - vertical fluctuations were largely suppressed and thus a $2 \mathrm{D}$ system was formed. Their number 
densities were statistically distributed over different compartments and no particle exchange between the subsystems occurred during the measurements. In the presence of an external magnetic field $B$, which was applied perpendicular to the sample plane, a magnetic moment $M(B)=\left(6.9 \times 10^{-11} \mathrm{~A} \mathrm{~m}^{2} / \mathrm{T}\right) B$ [14] was induced in the particles, which led to a repulsive magnetic dipole interaction $V_{i j}=\mu_{0}\left(M(B)^{2}\right) / 4 \pi r_{i j}^{3}$, where $r_{i j}$ denotes the distance between particles $i$ and $j[15,16]$ and $M(B)$ is described by the Langevin function. For convenience we introduce a dimensionless plasma parameter $\Gamma=$ $V^{\mathrm{mag}} / k_{B} T=\left(\mu_{0} M^{2}(\pi \rho)^{3 / 2}\right) /\left(4 \pi k_{B} T\right)$, where $\rho$ is the particle number density and $k_{B} T$ the thermal energy of the suspension $(T=295 \mathrm{~K})$. Accordingly, $B$ plays the role of an inverse effective temperature.

In order to create substrates with periodically arranged pinning sites, we imaged an array of laser tweezers to the center of one compartment inside the sample cell. This array was created by a diffractive optical element [17] which was illuminated with an expanded beam of a frequency doubled $\mathrm{Nd}: \mathrm{YVO}_{4}$ laser $(\lambda=532 \mathrm{~nm})$. The holographic image consisted of a square array of $9 \times 9$ about Gaussian light dots which differed in intensity only by a few percent [Fig. 1(a)]. The colloidal spheres were imaged with an inverted video microscope onto a CCD camera, and particle positions were determined online with a rate of $0.1 \mathrm{~Hz}$ and a lateral accuracy of $100 \mathrm{~nm}$. From these data we obtained particle trajectories, averaged particle densities, and pair correlation functions.

To characterize the light-induced pinning array, we measured the potential shape of the pinning sites as a function of the laser intensity. This was achieved by exposing the light pattern to a highly diluted colloidal suspension. From the probability distribution of the particles we obtained the laser potential acting on a particle by employing the Boltzmann statistics (inset of Fig. 1). Because the potential width amounts to about 5-6 $\mu \mathrm{m}$ which is much smaller than the mean particle distance, each pinning site is acting only to a single colloidal
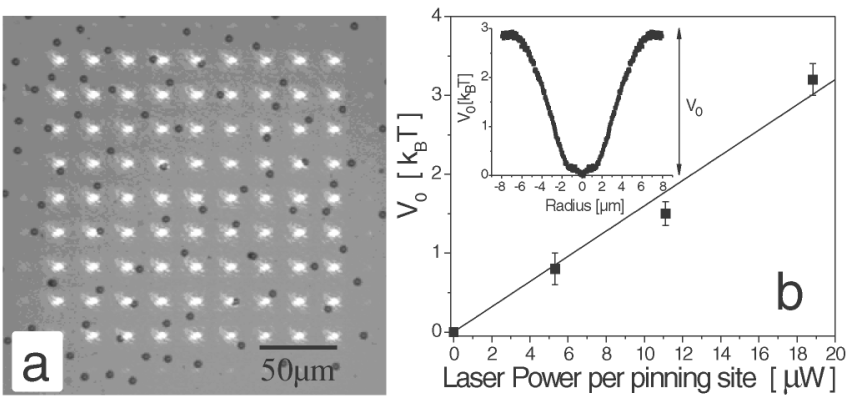

FIG. 1. (a) Photograph of a square $9 \times 9$ patterned holographic tweezer array (bright dots) and colloidal particles (dark objects). (b) Trapping strength of pinning sites as a function of the incident laser intensity. The inset displays the averaged potential shape of the pinning sites for $V_{0} \approx 3 k_{B} T$. particle. (This is in contrast to recent experiments where several particles were localized at pinning sites and colloidal molecules were formed [11]). As shown in Fig. 1, the pinning strength $V_{0}$ increases almost linearly as a function of the laser intensity, the latter being always in a range where thermal effects can be ruled out.

First, we investigated the effect of decreasing interparticle potential (i.e., plasma parameter $\Gamma$ ) at constant pinning strength $V_{0}=4.9 k_{B} T$. The particle density in the sample cell was kept constant and corresponded to about four particles per pinning site, i.e., a filling factor $m \approx 4$ (we observed a small increase of $m$ with decreasing $\Gamma$ due to the surrounding reservoir of particles). We measured the particle trajectories over about $3 \mathrm{~h}$ and also determined the averaged local particle density around a pinning site. Both quantities are plotted in Figs. 2(a)-2(f) as the left and right columns, respectively.

At the highest $B(\Gamma=65.3)$ used in this work, the colloidal interaction energy considerably exceeded the

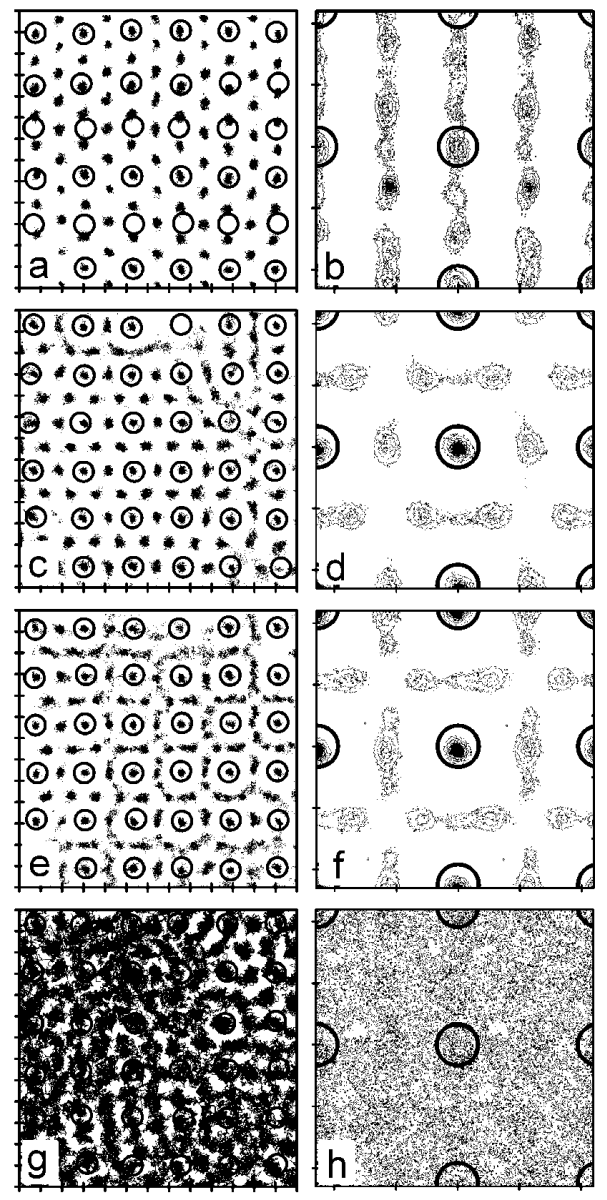

FIG. 2. Particle trajectories and contour plots for averaged local particle densities for $m \approx 4$ at different $\Gamma$ and $V_{0}$, respectively. (a),(b) $\Gamma=65.3, V_{0}=4.9 k_{B} T$; (c),(d) $\Gamma=37.6$, $V_{0}=4.9 k_{B} T$; (e),(f) $\Gamma=36.2, V_{0}=4.9 k_{B} T$; (g),(h) $\Gamma=23.5$, $V_{0}=1.5 k_{B} T$. The pinning sites are marked with open circles. The axis tics correspond to $10 \mu \mathrm{m}$ in all figures. 
influence of the substrate pinning sites. Therefore, the structure of the 2D colloidal system should be dominated by intercolloidal forces, i.e., a triangular lattice (in the absence of pinning sites crystallization takes place for $\Gamma \geq 56.5$ [18]). This is in good agreement with Fig. 2(a) where a triangular pattern is observed. A closer analysis, however, yields small deviations from an ideal triangular lattice by about $6 \%$ (regarding the length of the lattice vectors) which are due to the influence of the substrate pinning sites. While the colloidal pair potential favors a triangular lattice, as many as possible substrate pinning sites have to be occupied with a particle in order to minimize the free energy of the whole system. As shown in Figs. 2(a) and 2(b) the system responds by the alignment of a lattice vector of the colloidal crystal along one of the lattice vectors of the pinning array. While the periodicity of the substrate lattice is commensurate with the particle spacing in the horizontal direction in Fig. 2(a), there is a lattice mismatch in the vertical direction. On average only each second row of pinning sites is occupied with particles. Obviously, the interaction energy between the colloidal particles is too high to allow the system to lock to the periodicity of the substrate potential, and a floating or incommensurate solid is formed [19].

At $\Gamma=37.6$, the compressibility of the colloidal system increases and allows its geometry to accommodate to that of the substrate lattice. As shown in Figs. 2(c) and 2(d) almost all of the pinning sites are now occupied with a particle; i.e., the monolayer partially adopts the square symmetry of the substrate. The interstitial particles in this registered commensurate solid phase are arranged in a lattice with rhombic symmetry. This phase has also been observed in numerical calculations of the trajectories of a vortex lattice in the mixed state of type II superconductors in square periodic pinning arrays [3].

Further decrease of the particle interaction $(\Gamma=36.2)$ leads to a reduced localization of the interstitial particles while the particles at the pinning sites are still highly localized [Figs. 2(e) and 2(f)]. The trajectories of the interstitial particles are constrained to a squarelike grid which excludes the region around the pinning sites. This is due to the repulsive dipole interaction of the particles.

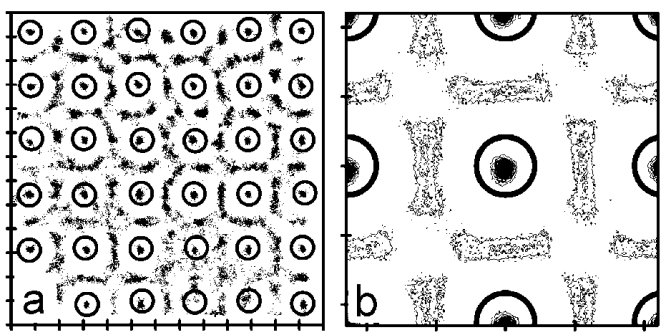

FIG. 3. Particle trajectories (a) and contour plot for averaged local particle densitiy (b) for $m \approx 4$ at $\Gamma=56.7$ and $V_{0}=$ $9.5 k_{B} T$. The pinning sites are marked with open circles. The axis tics correspond to $10 \mu \mathrm{m}$ in all figures.
Similar to the commensurate solid, this coexistence of localized and delocalized particles was also reported for vortex melting in periodic pinning arrays [3]. When both the strength of the particle interaction and the substrate pinning were further decreased $\left(\Gamma=23.5, V_{0}=1.5 k_{B} T\right)$, the particles at the pinning sites also become mobile and the entire lattice is in a liquid state as shown in Figs. 2(g) and $2(\mathrm{~h})$.

To understand the influence of interparticle and pinning forces onto the phase behavior, it is helpful to investigate to what extend $V_{0}$ affects the properties of the 2D colloidal system. Figures 3(a) and 3(b) show the trajectories and averaged particle density for $V_{0}=9.5 k_{B} T$ and a plasma parameter $\Gamma=56.7$ which is very close to the freezing transition of the particles on a flat substrate. Astonishingly, only the particles at the pinning sites are localized, whereas the interstitial particles are found to be rather mobile with their trajectories being confined to a square grid shape [similar to Figs. 2(e) and 2(f)]. It is interesting that for $V_{0}=4.9 k_{B} T$, i.e., weaker substrate pinning, a colloidal crystal was observed for $37.6<\Gamma<$ 65.3 [cf. Figs. 2(a)-2(d)]. Obviously, stronger localization of the particles at the pinning sites does not necessarily lead to crystallization even if the interaction among the colloids is increased. Because the effective interaction between particles at interstitial and regular lattice sites is enhanced by particle fluctuations, this might explain why an increased substrate strength leads to a less ordered structure. A similar observation, i.e., that melting can occur upon increased localization of particles, has been recently observed in 2D colloidal aggregates (trimers) which were confined to a triangular lattice by several interfering laser beams [11]. It has been demonstrated that these trimers can form colloidal molecular crystals with high positional and orientational order. When the localization of the trimers is too strong, however, their radial fluctuations decrease, which leads to a reduction of their effective interaction length and eventually to a loss of angular order [11]. It should be emphasized that the understanding of the role of particle fluctuations in the study discussed here needs further experimental and

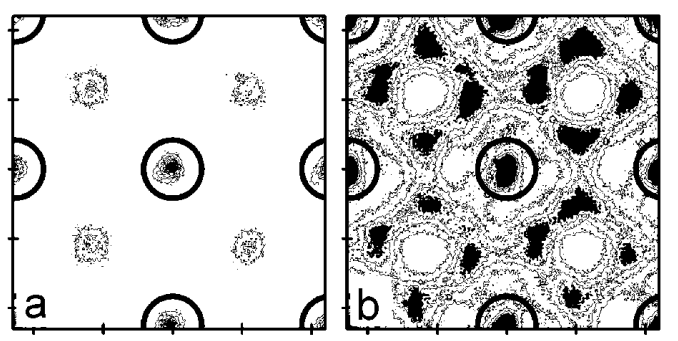

FIG. 4. Contour plots of averaged particle densities of crystalline structures for filling factors (a) $m \approx 2$ and (b) $m=5.5$. The pinning sites are marked with open circles. The axis tics correspond to $10 \mu \mathrm{m}$ in all figures. 
numerical investigations which are beyond the scope of this work.

Finally, we also investigated the colloidal structure for different filling factors $m$. Figure 4(a) shows the averaged local density of the commensurate crystalline phase for $m \approx 2, V_{0}=9.5 k_{B} T$, and $\Gamma=35$. The interstitial colloidal particles are localized in the center regions between the lattice sites, and the colloids form a centered $(1 \times 1)$ superlattice on the substrate. For $m=5.5$ the averaged local particle density of the interstitial particles is plotted for $V_{0}=6.4 k_{B} T$ and $\Gamma=44.4$ in Fig. 4(b). In contrast to Fig. 4(a), the particle density is almost zero at those positions where the interstitial particles were localized in Fig. 4(a). Now, the colloids are arranged in a squarelike geometry around these depletion zones. Because $m=$ 5.5 deviates slightly from perfect matching conditions, i.e., $m=5$, the localization of the interstitial particles is less pronounced and defect particles can diffuse along a squarelike network across the sample.

In general, we found the qualitative melting behavior to be independent of $m$ in case of square substrate potentials as investigated here (of course, the parameters where the melting occurs depends on the value of $m$ ). According to numerical simulations for vortices in superconductors $[3,4]$, however, this is true only for square pinning potentials. In case of triangular and kagome lattices and in case of noninteger $m$ values some particles (vortices) start to rotate around pinning sites. As $m$ is further increased (away from an integer value), the number of such ring excitations increases. Experimentally, triangular and kagome lattices can be generated by means of holographic gratings or interference patterns, and it will be an interesting question whether such a particle rotation can also be observed in colloidal systems. This would also allow one to study the role of defects in such systems (which are usually present in high-temperature superconductors) and to what extend they influence the phase behavior.

To summarize, we have experimentally investigated the phase behavior of 2D colloidal suspensions in the presence of periodic pinning arrays with square symmetry. Because of competing interplay of interparticle forces which favor a triangular symmetry of the colloids and the square substrate geometry, a rich phase behavior with incommensurate, commensurate, and liquid structures is observed. These results are in qualitative agreement with recent numerical studies on vortex melting in peri- odic pinning arrays. In addition, however, we also observed that within certain limits, stronger substrate potentials do not necessarily lead to an increased localization of interstitial particles. This effect is believed to be caused by particle fluctuations.

We thank C. Reichhardt for helpful discussions. This work was supported by the European Graduate College of the Deutsche Forschungsgemeinschaft.

[1] A. T. Fiory, A. F. Hebard, and S. Somekh, Appl. Phys. Lett. 32, 73 (1978).

[2] J. I. Martin, M. Velez, J. Nogues, and I. K. Schuller, Phys. Rev. Lett. 79, 1929 (1997).

[3] C. Reichhardt, C. J. Olson, R.T. Scalettar, and C.T. Zimányi, Phys. Rev. B 64, 144509 (2001).

[4] M. F. Laguna, C. A. Balseiro, D. Dominguez, and F. Nori, Phys. Rev. B 64, 104505 (2001).

[5] J. G. Dash, Films on Solid Surfaces (Academic Press, New York, San Francisco, London, 1975).

[6] S. K. Sinha, Ordering in Two Dimensions (Elsevier, New York, 1980).

[7] R. J. Birgeneau and P. M. Horn, Science 232, 329 (1986).

[8] I. Lyuksyutov, A. G. Naumovets, and V. Pokrovsky, TwoDimensional Crystals (Academic Press, New York, 1992).

[9] K.-H. Lin, J. C. Crocker, V. Prasad, A. Schofield, D. A. Weitz, T. C. Lubensky, and A. G. Yodh, Phys. Rev. Lett. 85, 1770 (2000).

[10] C. Bechinger, M. Brunner, and P. Leiderer, Phys. Rev. Lett. 86, 930 (2001).

[11] M. Brunner and C. Bechinger, Phys. Rev. Lett. 88, 248302 (2002).

[12] K. P. Velikov, C. G. Christova, R. P. A. Dullens, and A. v. Blaaderen, Science 296, 106 (2002).

[13] E. R. Dufresne and D. G. Grier, Rev. Sci. Instrum. 69, 1974 (1998).

[14] $M(B)$ was determined by comparing measured pair correlation functions $g(r)$ with simulations.

[15] R. Bubeck, S. Neser, C. Bechinger, and P. Leiderer, Prog. Colloid Polym. Sci. 110, 41 (1998).

[16] K. Zahn, J. M. Mendez, and G. Maret, Phys. Rev. Lett. 79, 175 (1997).

[17] Holoeye GmbH, Berlin, Germany.

[18] K. Zahn, R. Lenke, and G. Maret, Phys. Rev. Lett. 82, 2721 (1999).

[19] S. N. Coppersmith, D. S. Fisher, B. I. Halperin, P. A. Lee, and W. F. Brinkman, Phys. Rev. Lett. 46, 549 (1981). 